\title{
Angiosperm diversity of Borail Wildlife Sanctuary, Assam, India: Report - II
}

\author{
A. Kar', D. Boruah, P. Nath, N.K. Goswami and D. Saharia \\ The Energy and Resources Institute (TERI), North Eastern Regional Centre, Chachal, VIP Road, \\ Hengrabari, Guwahati-781 036, Assam, India \\ ${ }^{1}$ Corresponding author: E-mail: ashishvision20@gmail.com
}

[Received 02.06.2019; Revised \& accepted 26.06.2019; Published 30.06.2019]

\begin{abstract}
The present investigation deals with the composition of angiospermic plants in the Borail Wildlife Sanctuary, Assam. A total of 190 angiospermic species under 152 genera and 61 families were recorded from the sanctuary during the survey period. Angiosperm included 35 herbs, 30 shrubs, 59 trees, 27 climber, 7 lianas, 1 palm, 8 small grasses, 13 sedges, 2 bamboos, 3 canes, 4 epiphytic and 1 parasitic species.
\end{abstract}

Key words: Angiosperm diversity, Borail Wildlife Sanctuary

\section{INTRODUCTION}

Forests provide us with shelter, livelihoods, water, food and fuel security. All these activities directly or indirectly involve forests. Some are easy to figure out - fruits, paper and wood from trees, and so on. Others are less obvious, such as by-products that goes into everyday items like medicines, cosmetics and detergents. Forest provides ecosystem services that are critical to human welfare. They are serving as a buffer in natural disasters like flood and rainfalls, Absorbing harmful greenhouse gasses that produce climate change. Forest providing habitat both for water and land based species. Wildlife sanctuaries are helping to protect animals and safeguard their lives. Wildlife Sanctuaries help to protect animals from illegal activities, and serve as places where endangered animals breed and are therefore protected. It is a good place for animals where they can be protected (WWF 2018). Plants play an important role in wildlife sanctuary by providing shelter and food to the animals. Plants form the basic producer tier in any complex forested ecosystem.

The North-east Indian state Assam is lying in between $24^{\circ} 44^{\prime} \mathrm{N}$ to $27^{\circ} 45^{\prime} \mathrm{N}$ latitude and $89^{\circ} 41^{\prime}$ and $96^{\circ} 02^{\prime} \mathrm{E}$ longitudes and has an geographical area of $78,433 \mathrm{sq} \mathrm{km}$. The climatic condition and wide variety in physical features witnessed in Assam have resulted in a diversity of ecological habitats such as forests, grasslands, wetlands, which harbour and sustain wide ranging floral and faunal species. The state is surrounded by hills and mountains on the north, east and the south side. The state has the Brahmaputra valley in the northern part bordering to Arunachal Pradesh and the Barak valley in the southern part bordering to Mizoram, Tripura and Meghalaya. The state is enriched with 3832 (dicotyledons 2752 and monocotyledon 1080) species of angiosperms comprising of 1370 (dicotyledons 1012 and monocotyledon 368) genera distributed in 229 (dicotyledons 189 and monocotyledon 40) families (Chowdhury 2005). 
The Barail Wildlife Sanctuary (BWS) is located between latitude $24^{\circ} 58^{\prime}-25^{\circ} 50^{\prime} \mathrm{N}$ and longitude $92^{\circ} 50^{\prime}-92^{\circ} 52^{\prime} \mathrm{E}$. The sanctuary is situated in the northern part of Cachar district of Assam and lies along the foothills of the North Cachar and Barail hills (PLATE I). The total area covered by this wildlife sanctuary is $326.24 \mathrm{sq} \mathrm{km}$. Barail Wildlife Sanctuary is a combination Barail Reserve Forest and North Cachar Reserve Forest, which were together upgraded to Barail Wildlife Sanctuary in June 2004. The climate is humid tropical to sub-tropical; annual rainfall varies from 200 to $600 \mathrm{~cm}$ and the average humidity is $72-90$ $\%$. Temperatures range minimum $8^{\circ} \mathrm{C}$ and maximum temperatures $38^{\circ} \mathrm{C}$. Rivers like Jatinga, Daloo (Photo -14), Kayong, Gumra, and Boleswar has formed the riverine forest of BWS. The sanctuary is characterized by undulating hills having altitudinal range of less than $30 \mathrm{~m}$ to more than $1867 \mathrm{~m}$. As per classification of Champion and Seth (1968), the sanctuary has 2 broad groups of forests, tropical wet evergreen (Photo -15) and tropical semi-evergreen forests. Tree, shrub and liana species (Photo - 13) forming a thick vegetation of the sanctuary. Forest floor is enriched by many herbaceous species. The main secondary landscape elements are cultivated flatland, extensive bamboo brakes, tree plantations (Teak and Sal), secondary and disturbed forest (betel-vine plantation), and village gardens including Areca nut plantations (Barbhuiya \& Singh 2012; Hussain 2015; Bora \& Bhattacharyya 2017; Bora et al. 2017).

Several publications came out on assessment of plant diversity in protected areas of Assam. Some of them are Begum et al. (2011) on sketch of the flora of Nameri National Park; Sarkar \& Devi (2015) on Assessment of plant diversity in Hollongapar Gibbon Wildlife Sanctuary; Jain \& Hajra (1975) on the plant diversity of Manas Wildlife Sanctuary; Dutta et al. (1974) on forest flora of North Cachar Hills and Borail Range; Buragohain \& Swargiari (2016) on Diversity and conservation of Ficus L. (Moraceae) in Chakrashila Wildlife Sanctuary; Baruah \& Baruah (2000) on hydrophytes of Kaziranga National Park; Baruah \& Baruah (2007) on vegetation characteristics of grassland of Kaziranga National Park ; Baruah et al. (2003) on biodiversity status in Manas Biosphere reserve; Bharali \& Borua (2003) on diversity of orchid flora of Dibru-Saikhowa National Park and Biosphere Reserve; Gogoi et al. (2009) on orchid flora of Joypur Reserve Forest of Dibrugarh district; Gogoi et al. (2010) on orchid flora of Dibru-Saikhowa National Park and Biosphere Reserve; Gogoi (2005) on the genus Dendrobium in Dibru-Saikhowa National park and Biosphere Reserve; Dey et al. (2007) on Orchid diversity in Manas National Park, Assam; Bujarbarua \& Sarma (2006) on the diversity of Poaceae in Gibbon Wildlife Sanctuary, Assam; Konwar et al. (2009) on the abundance of food plant species and food habits of Rhinoceros unicornis L. in Pobitora Wildlife Sanctuary; Talukdar \& Deori (2017) on Floristic Diversity of Laokhowa Wildlife Sanctuary; Nath (2012) on Aquatic macrophytes of Laokhowa Wildlife Sanctuary; Deori \& Talukdar (2015) Floristic Diversity of Barnadi Wildlife Sanctuary; Kar et al. (2015) on vascular plant diversity in Amchang Wildlife Sanctuary; and Hajra \& Jain (1996) on plants of Kaziranga and Manas.

As far as plant diversity study of the Borail Wildlife Sanctuary is concerned, only few report on angiosperm plants (Dutta et al. 1974) is available. Bora \& Bhattacharyya (2017) worked on the trees and lianas of Borail Wildlife Sanctuary; Bora et al. (2017) studied the Grasses and bamboos of Barail Wildlife Sanctuary are available. Kar et al. (2018) carried out some studies on angiosperm flora of the sanctuary and recorded 281 angiosperm species out of which 224 species were dicots and 57 species were monocots. Considering the possibilities to add more species in the angiosperm category from this sanctuary, thus this work was taken up to provide information on the occurrence of more species of angiosperms in BWS. Therefore, the present investigations were carried out with the objective of record previously unrecorded angiospermic plants of the Borail wildlife Sanctuary. 


\section{MATERIALS AND METHODS}

Surveys of the angiosperm plants of Borail Wildlife Sanctuary (Figure 1) were conducted from November 2018 to May 2019 covering both wild and cultivated species within the sanctuary. Surveys were conducted from fifteen sites of the sanctuary viz., Malidor, Kalaincherra, Durganagar, Lakhicherra, Gumra, Madhura, Indranagar, Khasia Punji, Hmar Punji, Kapocherra, Balacherra, Marwa cherra, Durbin tilla, Damcherra, Bandarkhal and dense forest areas. External mor-phological characteristics including hairs, stipules and floral parts, of collected specimens was observed using Foldscope microscope. Characteristics of each species were observed and noted. The collected specimens were processed into mounted herbarium specimen following standard herbarium techniques (Jain \& Rao 1977). Identification of the specimens was done by comparing the field descriptions and observations with the descriptions available in authentic literature (Kanjilal et al. 1934-1940; Chowdhury 2005) and confirmed by matching at ASSAM Herbarium. Herbarium specimens were deposited at the TERI herbarium for future reference. The International Plant Name Index (IPNI 2012), The Plant List (2013) and Tropicos (2017) were consulted for current nomenclature and family delimitation of all recorded taxa. The Angiosperm Phylogeny Group III Classifica-tion (APG III 2009) was followed for the classification of families. The families were arranged in alphabetical order.

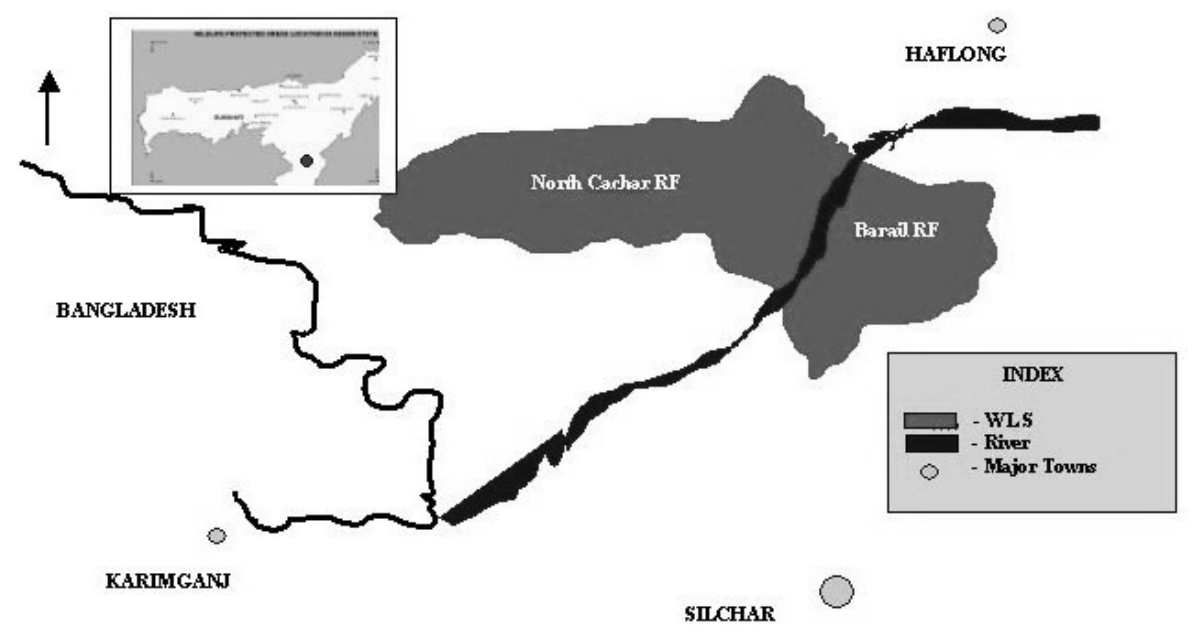

Figure 1. Location map of Borail Wildlife Sanctuary (not in scale)

\section{RESULTS AND DISCUSSION}

Study recorded total 190 species under 154 genera from 63 families (Table 1) were recorded from the study area. Angiosperm comprises of 63 families (dicot 48 and monocot 15), 154 genera (dicot 110 and monocot 44) and 190 species (dicot 135 and monocot 55). Out of the total recorded 190 angiosperm species the composition were 35 herbs, 30 shrubs, 59 trees, 27 climbers, 7 liana, 1 palm, 8 small grasses, 13 sedges, 2 bamboo, 3 canes, 4 epiphytic and 1 parasitic species (Figure 2).

Among the dicotyledons, Leguminosae is the most dominant family in the study area in respect to number of species with 23 species, followed by Malvaceae, Meliaceae and Rubiaceae each with 8 species, and Menispermaceae ( 5 species) etc. In Monocots Cyperaceae is the dominant family with 13 species followed by Poaceae with 10 species, Araceae with 8 species, Orchidaceae with 5 species, and Commeliniaceae with 4 species. Among the dicotyledons 
Table 1. Angiosperm plant of Borail Wildlife Sanctuary

[Abbreviations used: Habit: $\mathrm{H}=$ Herb; $\mathrm{S}=$ Shrub $\mathrm{Cl}=$ Climber; $\mathrm{L}=$ Lianas; $\mathrm{T}=$ Tree $; \mathrm{PA}=\mathrm{Palm} ; \mathrm{G}=$ Grass; $\mathrm{SE}=$ Sedge; $\mathrm{B}=$ Bamboo; $\mathrm{EP}=$ Epiphytic $; \mathrm{P}=$ Parasitic $; \mathrm{CA}=$ Cane. Habitat: $\mathrm{RS}=$ Road sides; $\mathrm{SS}=$ Stream side $; \mathrm{HS}=$ Hill slopes $; \mathrm{RB}=$ River bank $; \mathrm{F}=$ Forest GL $=$ Grassland $]$

\begin{tabular}{|c|c|c|c|c|}
\hline SI No & Name of the plants & Habit & Habitat & Distribution \\
\hline & \multicolumn{4}{|c|}{ DICOTYLEDONS } \\
\hline & Anacardiaceae & & & \\
\hline \multirow[t]{2}{*}{1} & Mangifera sylvatica Roxb. & $\mathrm{T}$ & $\mathrm{F}$ & Rare \\
\hline & Annonaceae & & & \\
\hline \multirow[t]{2}{*}{2} & Cananga odorata (Lam.) Hook.f. \& Thomson & $\mathrm{T}$ & $\mathrm{F}$ & Rare \\
\hline & Apocynaceae & & & \\
\hline 3 & Holarrhena pubescens Wall. ex G.Don & $\mathrm{T}$ & $\mathrm{F}$ & Common \\
\hline \multirow[t]{2}{*}{4} & $\begin{array}{l}\text { Hoya acuminata (Wight) Benth. ex Hook.f. } \\
\text { [Photo 22 ] }\end{array}$ & $\mathrm{Cl}$ & $\mathrm{F}$ & Common \\
\hline & Balsaminaceae & & & \\
\hline \multirow[t]{2}{*}{5} & Impatiens trilobata Colebr. & $\mathrm{H}$ & SS & Rare \\
\hline & Begoniaceae & & & \\
\hline \multirow[t]{2}{*}{6} & Begonia thomsonii A.DC. [ Photo: 24 ] & $\mathrm{H}$ & SS & Rare \\
\hline & Burseraceae & & & \\
\hline 7 & Bursera serrata Wall. ex Colebr. & $\mathrm{T}$ & $\mathrm{F}$ & Rare \\
\hline 8 & Canarium bengalense Roxb. & $\mathrm{T}$ & $\mathrm{F}$ & Rare \\
\hline 9 & Garuga floribunda Decne. & $\mathrm{T}$ & $\mathrm{F}$ & Common \\
\hline \multirow[t]{2}{*}{10} & Garuga pinnata Roxb. & $\mathrm{T}$ & $\mathrm{F}$ & Rare \\
\hline & Cannabaceae & & & \\
\hline 11 & Holoptelea integrifolia Planch. & $\mathrm{T}$ & $\mathrm{F}$ & Rare \\
\hline 12 & Trema orientalis (L.) Blume & $\mathrm{T}$ & $\mathrm{F}$ & Rare \\
\hline \multirow[t]{2}{*}{13} & Trema tomentosa (Roxb.) H.Hara & $\mathrm{T}$ & $\mathrm{F}$ & Rare \\
\hline & Calophyllaceae & & & \\
\hline \multirow[t]{2}{*}{14} & Mesua ferrea $\mathrm{L}$. & $\mathrm{T}$ & $\mathrm{F}$ & Rare \\
\hline & Clusiaceae & & & \\
\hline 15 & $\begin{array}{l}\text { Garcinia xanthochymus Hook.f. ex } \\
\text { T.Anderson }\end{array}$ & $\mathrm{T}$ & $\mathrm{F}$ & Very rare \\
\hline \multirow[t]{2}{*}{16} & Kayea floribunda Wall. & $\mathrm{T}$ & $\mathrm{F}$ & Very rare \\
\hline & Compositae/ Asteraceae & & & \\
\hline 17 & $\begin{array}{l}\text { Acmella paniculata (Wall. ex DC.) R.K. } \\
\text { Jansen }\end{array}$ & $\mathrm{H}$ & $\mathrm{RS}$ & Common \\
\hline 18 & Blumea balsamifera (L.) DC. & $\mathrm{H}$ & HS & Rare \\
\hline \multirow[t]{2}{*}{19} & Galinsoga parviflora Cav. & $\mathrm{H}$ & $\mathrm{RS}$ & Common \\
\hline & Convolvulaceae & & & \\
\hline 20 & Ipomoea quamoclit $\mathrm{L}$. & $\mathrm{Cl}$ & $\mathrm{RS}$ & Common \\
\hline 21 & Merremia vitifolia (Burm.f.) Hallier.f. & $\mathrm{Cl}$ & $\mathrm{RS}$ & Common \\
\hline
\end{tabular}


Angiosperms of Borail Wildlife Sanctuary: II

\begin{tabular}{|c|c|c|c|c|}
\hline SI No & Name of the plants & Habit & Habitat & Distribution \\
\hline \multirow[t]{2}{*}{22} & Poranopsis paniculata (Roxb.) Roberty & $\mathrm{Cl}$ & $\mathrm{RS}$ & Common \\
\hline & Cornaceae & & & \\
\hline \multirow[t]{2}{*}{23} & Alangium chinense (Lour.) Harms & $\mathrm{T}$ & $\mathrm{F}$ & Rare \\
\hline & Cucurbitaceae & & & \\
\hline 24 & Diplocyclos palmatus (L.) C. Jeffrey & $\mathrm{Cl}$ & $\mathrm{F}$ & Rare \\
\hline 25 & Indofevillea khasiana Chattarjee & $\mathrm{Cl}$ & $\mathrm{F}$ & Rare \\
\hline 26 & Mukia maderaspatana (L.) M. Roem. & $\mathrm{Cl}$ & HS & Rare \\
\hline \multirow[t]{2}{*}{27} & Solena amplexicaulis (Lam.) Gandhi & $\mathrm{Cl}$ & $\mathrm{F}$ & Rare \\
\hline & Dilleniaceae & & & \\
\hline 28 & Dillenia indica $\mathrm{L}$. & $\mathrm{T}$ & $\mathrm{F}$ & Common \\
\hline \multirow[t]{2}{*}{29} & Dillenia pentagyna Roxb. & $\mathrm{T}$ & $\mathrm{F}$ & Very rare \\
\hline & Dipterocarpaceae & & & \\
\hline \multirow[t]{2}{*}{30} & Dipterocarpus retusus Blume & $\mathrm{T}$ & $\mathrm{F}$ & Rare \\
\hline & Elaeocarpaceae & & & \\
\hline \multirow[t]{2}{*}{31} & $\begin{array}{l}\text { Sloanea tomentosa (Benth.) Rehder \& } \\
\text { E.H.Wilson }\end{array}$ & $\mathrm{T}$ & $\mathrm{F}$ & Very rare \\
\hline & Fagaceae & & & \\
\hline \multirow[t]{2}{*}{32} & $\begin{array}{l}\text { Lithocarpus elegans (Blume) Hatus ex } \\
\text { Soepadmo }\end{array}$ & $\mathrm{T}$ & $\mathrm{F}$ & Very rare \\
\hline & Icacinaceae & & & \\
\hline 33 & $\begin{array}{l}\text { Natsiatum herpeticum Buch.-Ham. ex Arn. } \\
\text { [Photo } 23 \text { ] }\end{array}$ & $\mathrm{Cl}$ & $\mathrm{F}$ & Common \\
\hline \multirow[t]{2}{*}{34} & Nothapodytes nimmoniana (J. Graham) Mabb. & $\mathrm{S}$ & $\mathrm{F}$ & Very rare \\
\hline & Juglandaceae & & & \\
\hline \multirow[t]{2}{*}{35} & Engelhardtia spicata Lechen ex Blume & $\mathrm{T}$ & $\mathrm{F}$ & Rare \\
\hline & Lamiaceae/ Labiatae & & & \\
\hline 36 & $\begin{array}{l}\text { Gomphostemma parviflorum Wall. ex Benth. } \\
\text { [Photo 21] }\end{array}$ & $\mathrm{S}$ & $\mathrm{F}$ & Very Rare \\
\hline 37 & Pogostemon benghalensis (Burm.f.) Kuntze & $\mathrm{S}$ & RS & Rare \\
\hline 38 & Premna mollissima Roth & $\mathrm{T}$ & $\mathrm{F}$ & Common \\
\hline \multirow[t]{2}{*}{39} & Premna longifolia Roxb. & $\mathrm{T}$ & $\mathrm{F}$ & Common \\
\hline & Lauraceae & & & \\
\hline 40 & $\begin{array}{l}\text { Cinnamomum tamala (Buch.-Ham.) T. Nees \& } \\
\text { Eberm. }\end{array}$ & $\mathrm{T}$ & $\mathrm{F}$ & Common \\
\hline 41 & Cinnamomum verum J.Presl. & $\mathrm{T}$ & $\mathrm{F}$ & Very Rare \\
\hline 42 & Litsea monopetala (Roxb.) Pers [Photo 11] & $\mathrm{T}$ & $\mathrm{F}$ & Very Rare \\
\hline \multirow[t]{2}{*}{43} & Litsea nitida (Roxb.) Hook.f. & $\mathrm{T}$ & $\mathrm{F}$ & Common \\
\hline & Leguminosae/ Fabaceae & & & \\
\hline 44 & Acacia caesia (L.) Willd. [Photo 3] & $\mathrm{L}$ & $\mathrm{F}$ & Common \\
\hline 45 & Acacia myriophylla Graham & $\mathrm{T}$ & $\mathrm{F}$ & Common \\
\hline
\end{tabular}




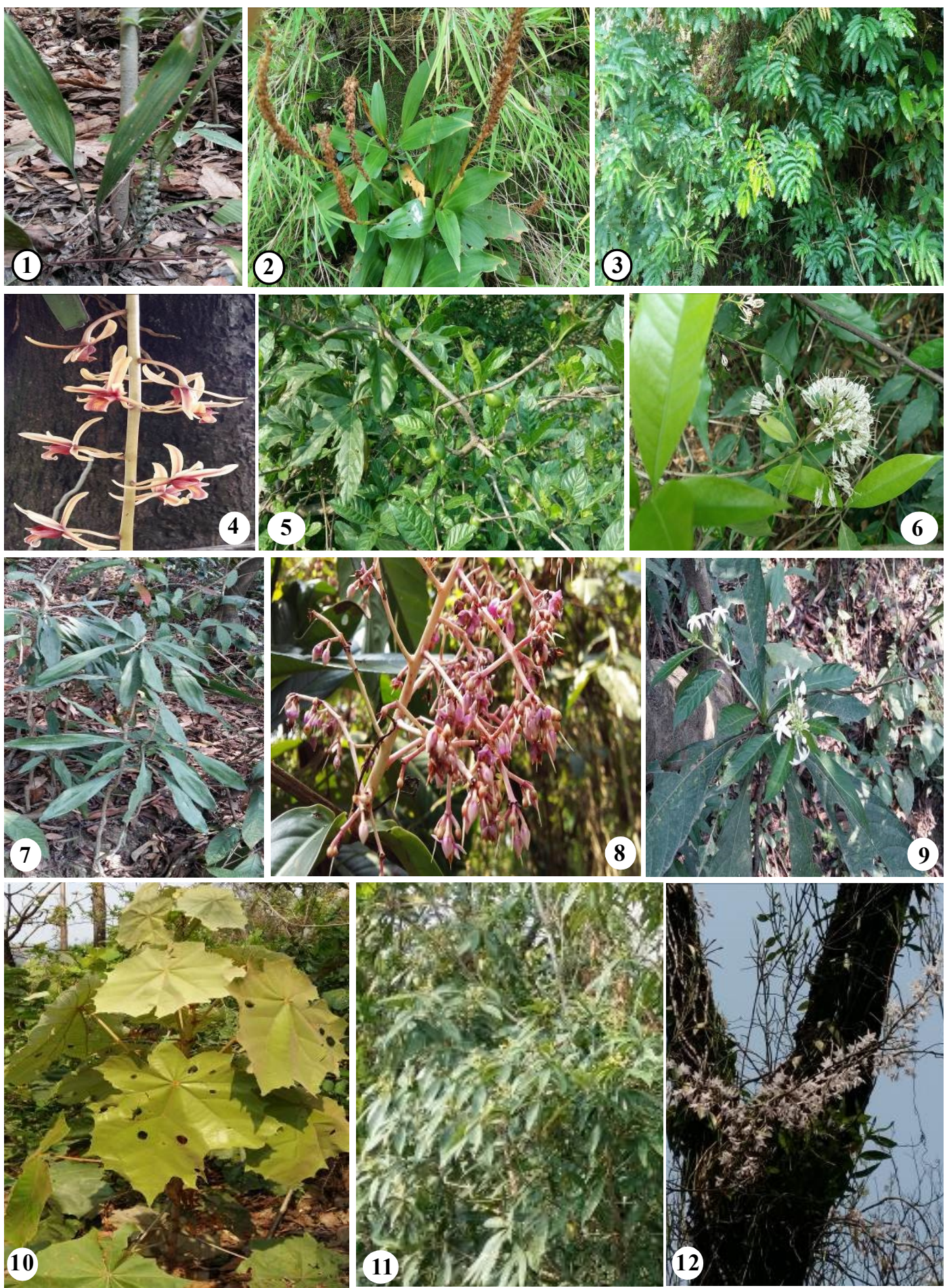

PLATE - I: 1. Peliosanthes teeta; 2. Liparis elliptica; 3 Acacia caesia ; 4. Cymbidium aloifolium; 5. Catunaregum spinosa ; 6. Ixora rangonensis ; 7. Dracaena angustifolia; 8. Ardisia paniculata ; 9. Morinda angustifolia; 10. Pterospermum acerifolium; 11. Litsea monopetala; 12. Dendrobium lituiflorum 


\begin{tabular}{|c|c|c|c|c|}
\hline SI No & Name of the plants & Habit & Habitat & Distribution \\
\hline 46 & Acrocarpus fraxinifolius Arn. & $\mathrm{T}$ & $\mathrm{F}$ & Rare \\
\hline 47 & Bauhinia racemosa Lam. & $\mathrm{S}$ & $\mathrm{F}$ & Rare \\
\hline 48 & Chamaecrista mimosoides (L.) Greene & $\mathrm{S}$ & $\mathrm{RS}$ & Rare \\
\hline 49 & Caesalpinia cucullata Roxb. & $\mathrm{L}$ & $\mathrm{F}$ & Rare \\
\hline 50 & Caesalpinia tortuosa Roxb. & $\mathrm{Cl}$ & $\mathrm{F}$ & Rare \\
\hline 51 & Crotalaria pallida Aiton & $\mathrm{S}$ & $\mathrm{RS}$ & Common \\
\hline 52 & Crotalaria juncea $\mathrm{L}$. & $\mathrm{S}$ & RS & Rare \\
\hline 53 & Derris elliptica (Wall.) Benth. & $\mathrm{S}$ & SS & Rare \\
\hline 54 & Dalbergia pinnata Lour. Prain & $\mathrm{S}$ & $\mathrm{RB}$ & Rare \\
\hline 55 & Derris scandens (Roxb.) Benth. & $\mathrm{Cl}$ & RB & Rare \\
\hline 56 & Caesalpinia bonduc (L) Roxb. & $\mathrm{L}$ & RS & Very rare \\
\hline 57 & Moullava spicta (Dalzell) Nicolson & $\mathrm{L}$ & $\mathrm{F}$ & Rare \\
\hline 58 & Mucuna bracteata DC. & $\mathrm{Cl}$ & RS & Rare \\
\hline 59 & Ohwia caudate (Thunb.) H.Ohashi & $\mathrm{S}$ & $\mathrm{F}$ & Rare \\
\hline 60 & Peltophorum pterocarpum (DC.) K. Heyne & $\mathrm{T}$ & $\mathrm{F}$ & Common \\
\hline 61 & Saraca asoca (Roxb).Willd. & $\mathrm{T}$ & $\mathrm{F}$ & Very rare \\
\hline 62 & Senna hirsuta (L.) Irwin \& Barneby & $\mathrm{S}$ & RS & Common \\
\hline 63 & Senna siamea (Lam.) H.S. Irwin et Barneby & $\mathrm{T}$ & $\mathrm{F}$ & Common \\
\hline 64 & Senegalia pennata (L.) Maslin & $\mathrm{L}$ & $\mathrm{F}$ & Common \\
\hline 65 & Tamarindus indica $\mathrm{L}$. & $\mathrm{T}$ & $\mathrm{F}$ & Common \\
\hline 66 & Tephrosia candida (Roxb.) DC. & $\mathrm{S}$ & RS & Rare \\
\hline \multirow[t]{2}{*}{67} & Uraria picta (Jacq.) DC. & $\mathrm{H}$ & $\mathrm{F}$ & Very Rare \\
\hline & Loranthaceae & & & \\
\hline \multirow[t]{2}{*}{68} & Tolypanthus involucratus Blume & $\mathrm{P}$ & $\mathrm{F}$ & Very common \\
\hline & Magnoliaceae & & & \\
\hline 69 & $\begin{array}{l}\text { Magnolia hodgsonii (Hook.f. \& Thomson) H. } \\
\text { Keng }\end{array}$ & $\mathrm{T}$ & $\mathrm{F}$ & Very Rare \\
\hline 70 & Magnolia pterocarpa Roxb. & $\mathrm{T}$ & $\mathrm{F}$ & Very Rare \\
\hline \multirow[t]{2}{*}{71} & Magnolia champaca (L.) Baill.ex Pierre & $\mathrm{T}$ & $\mathrm{F}$ & Rare \\
\hline & Malvaceae & & & \\
\hline 72 & Abelmoschus manihot (L.) Medik. & $\mathrm{S}$ & RS & Rare \\
\hline 73 & Byttneria aspera Collebr.ex Wall. & $\mathrm{L}$ & $\mathrm{F}$ & Rare \\
\hline 74 & Corchorus aestuans $\mathrm{L}$. & $\mathrm{H}$ & GL & Common \\
\hline 75 & Grewia abutilifolia Vent. ex Juss. & $\mathrm{S}$ & $\mathrm{RS}$ & Common \\
\hline 76 & Grewia eriocarpa Juss. & $\mathrm{S}$ & $\mathrm{RS}$ & Common \\
\hline 77 & Grewia serrulata DC. & $\mathrm{S}$ & $\mathrm{RS}$ & Common \\
\hline 78 & Hibiscus aculeatus Walter & $\mathrm{H}$ & RS & Rare \\
\hline 79 & $\begin{array}{l}\text { Pterospermum acerifolium (L.) Willd. } \\
\text { [Photo 10] }\end{array}$ & $\mathrm{T}$ & $\mathrm{F}$ & Common \\
\hline 80 & Pterospermum javanicum Jungh & $\mathrm{T}$ & $\mathrm{F}$ & Rare \\
\hline 81 & Sida spinosa L. & $\mathrm{H}$ & $\mathrm{RS}$ & Rare \\
\hline
\end{tabular}


A. Kar et al. 129

\begin{tabular}{|c|c|c|c|c|}
\hline SI No & Name of the plants & Habit & Habitat & Distribution \\
\hline 82 & Sterculia guttata Roxb. & $\mathrm{T}$ & $\mathrm{F}$ & Common \\
\hline \multirow[t]{2}{*}{83} & Triumfetta annua $\mathrm{L}$. & $\mathrm{H}$ & RS & Very common \\
\hline & Meliaceae & & & \\
\hline 84 & Aglaia cucullata (Roxb.) Pellegr & $\mathrm{T}$ & $\mathrm{F}$ & Rare \\
\hline 85 & Aglaia edulis (Roxb.) Wall. & $\mathrm{T}$ & $\mathrm{F}$ & Rare \\
\hline 86 & Aglaia perviridis Hiern. & $\mathrm{T}$ & $\mathrm{F}$ & Common \\
\hline 87 & Aglaia spectabilis (Miq.) S.S. Jain \& S.Bennet & $\mathrm{T}$ & $\mathrm{F}$ & Rare \\
\hline 88 & Chisocheton cumingianus (C.DC.) Harms & $\mathrm{T}$ & $\mathrm{F}$ & Rare \\
\hline 89 & Dysoxylum cauliflorum Hiern & $\mathrm{T}$ & $\mathrm{F}$ & Rare \\
\hline 90 & Dysoxylum grande Hiern & $\mathrm{T}$ & $\mathrm{F}$ & Common \\
\hline \multirow[t]{2}{*}{91} & Dysoxylum excelsum Blume & $\mathrm{T}$ & $\mathrm{F}$ & Common \\
\hline & Menispermaceae & & & \\
\hline 92 & Anamirta cocculus (L.) Wight \& Arn. & $\mathrm{Cl}$ & $\mathrm{F}$ & Common \\
\hline 93 & Cocculus orbicalutus (L.) DC. & $\mathrm{Cl}$ & $\mathrm{F}$ & Common \\
\hline 94 & $\begin{array}{l}\text { Haematocarpus validus (Miers) Bakh.f. ex } \\
\text { Forman }\end{array}$ & $\mathrm{Cl}$ & HS & Rare \\
\hline 95 & Hypserpa nitida Miers. ex Benth. & $\mathrm{Cl}$ & HS & Rare \\
\hline 96 & Parabaena sagittata Miers. & $\mathrm{Cl}$ & $\mathrm{F}$ & Rare \\
\hline 97 & Pericampylus glaucus (Lam.) Merr. & $\mathrm{Cl}$ & $\mathrm{F}$ & Rare \\
\hline \multirow[t]{2}{*}{98} & Tinomiscium petiolare Hook.f. \& Thomson & $\mathrm{L}$ & HS & Rare \\
\hline & Moraceae & & & \\
\hline \multirow[t]{2}{*}{99} & Ficus tinctoria G. Forst. [Photo 20] & $\mathrm{T}$ & RS & Rare \\
\hline & Myrtaceae & & & \\
\hline \multirow[t]{2}{*}{100} & Syzygium fruticosum DC. & $\mathrm{T}$ & $\mathrm{F}$ & Common \\
\hline & Oxalidaceae & & & \\
\hline 101 & Biophytum sensitivum (L.) DC. & $\mathrm{H}$ & $\mathrm{RS}$ & Rare \\
\hline \multirow[t]{2}{*}{102} & Oxalis debilis Kunth & $\mathrm{H}$ & RS & Rare \\
\hline & Passifloraceae & & & \\
\hline \multirow[t]{2}{*}{103} & Passiflora foetida $\mathrm{L}$. & $\mathrm{Cl}$ & $\mathrm{RS}$ & Very rare \\
\hline & Phyllanthaceae & & & \\
\hline 104 & Bischofia javanica Blume [Photo 16] & $\mathrm{T}$ & RS & Very rare \\
\hline \multirow[t]{2}{*}{105} & Bridelia retusa (L.) A. Juss & $\mathrm{T}$ & $\mathrm{F}$ & Rare \\
\hline & Piperaceae & & & \\
\hline \multirow[t]{2}{*}{106} & $\begin{array}{l}\text { Piper berembunse A.Chaveerach \& R } \\
\text { Sudmoon [Photo 17] }\end{array}$ & $\mathrm{Cl}$ & $\mathrm{F}$ & Very rare \\
\hline & Primulaceae & & & \\
\hline \multirow[t]{2}{*}{107} & Ardisia paniculata (Roxb.) [Photo 8] & $\mathrm{S}$ & $\mathrm{F}$ & Very rare \\
\hline & Rhamnaceae & & & \\
\hline \multirow[t]{2}{*}{108} & Gouania leptostachya DC. & $\mathrm{Cl}$ & $\mathrm{F}$ & Very rare \\
\hline & Rhizophoraceae & & & \\
\hline 109 & Carallia brachiata (Lour.) Merr. & $\mathrm{T}$ & $\mathrm{F}$ & Common \\
\hline
\end{tabular}




\begin{tabular}{|c|c|c|c|c|}
\hline SI No & Name of the plants & Habit & Habitat & Distribution \\
\hline & Rubiaceae & & & \\
\hline 110 & $\begin{array}{l}\text { Catunaregam spinosa (Thunb.) Triveng. } \\
\text { [Photo : 5] }\end{array}$ & $\mathrm{S}$ & $\mathrm{F}$ & Rare \\
\hline 111 & Coffea benghalensis B. Heyne ex Schult. & $\mathrm{H}$ & $\mathrm{F}$ & Common \\
\hline 112 & Ceriscoides campanulata (Roxb.) Triveng. & $\mathrm{S}$ & $\mathrm{F}$ & Rare \\
\hline 113 & $\begin{array}{l}\text { Dentella repens var.serpyllifolia (Wall. ex } \\
\text { Craib) Verdc. }\end{array}$ & $\mathrm{H}$ & $\mathrm{RS}$ & Common \\
\hline 114 & Ixora rangonensis Bremek [Photo 6] & $\mathrm{S}$ & $\mathrm{F}$ & Very rare \\
\hline 115 & Meyna laxiflora Robyns & $\mathrm{S}$ & RS & Common \\
\hline 116 & Morinda angustifolia Roxb. [Photo 9] & $\mathrm{S}$ & $\mathrm{F}$ & Very rare \\
\hline \multirow[t]{2}{*}{117} & Spermacoce alata Aubl. & $\mathrm{H}$ & GL & Very common \\
\hline & Rutaceae & & & \\
\hline 118 & Clausena heptaphylla (Roxb.) Wight \& Arn. & $\mathrm{S}$ & $\mathrm{F}$ & Rare \\
\hline 119 & Murraya koenigii (L.) Spreng. & $\mathrm{S}$ & $\mathrm{F}$ & Very common \\
\hline 120 & Murraya paniculata (L.) Jack & $\mathrm{S}$ & $\mathrm{F}$ & Rare \\
\hline 121 & Toddalia asiatica (L.) Lam. & $\mathrm{Cl}$ & $\mathrm{F}$ & Very rare \\
\hline \multirow[t]{2}{*}{122} & Zanthoxylum nitidum (Roxb.) DC. & $\mathrm{Cl}$ & $\mathrm{F}$ & Rare \\
\hline & Salicaceae & & & \\
\hline 123 & Casearia glomerata Roxb. & $\mathrm{T}$ & $\mathrm{F}$ & Rare \\
\hline \multirow[t]{2}{*}{124} & Xylosma longifolia Clos & $\mathrm{T}$ & $\mathrm{F}$ & Rare \\
\hline & Sabiaceae & & & \\
\hline 125 & Meliosma pinnata (Roxb.) Maxim. & $\mathrm{T}$ & SS & Rare \\
\hline \multirow[t]{2}{*}{126} & Meliosma simplicifolia (Roxb.) Walp. & $\mathrm{T}$ & $\mathrm{F}$ & Rare \\
\hline & Simaroubaceae & & & \\
\hline 127 & Ailanthus altissima (Mill.) Swingle & $\mathrm{T}$ & $\mathrm{F}$ & Very rare \\
\hline 128 & Ailanthus excelsa Roxb. & $\mathrm{T}$ & $\mathrm{F}$ & Very rare \\
\hline \multirow[t]{2}{*}{129} & Ailanthus integrifolia Lam. & $\mathrm{T}$ & $\mathrm{F}$ & Very rare \\
\hline & Solanaceae & & & \\
\hline \multirow[t]{2}{*}{130} & Cestrum nocturnum L. & $\mathrm{S}$ & $\mathrm{RS}$ & Very common \\
\hline & Tetramelaceae & & & \\
\hline \multirow[t]{2}{*}{131} & Tetrameles nudiflora R.Br. & $\mathrm{T}$ & $\mathrm{F}$ & Rare \\
\hline & Urticaceae & & & \\
\hline 132 & Boehmeria glomerulifera Miq. & $\mathrm{S}$ & SS & Common \\
\hline \multirow[t]{2}{*}{133} & Pilea scripta (Buch.- Ham. ex D.Don)Wedd. & $\mathrm{S}$ & SS & Common \\
\hline & Vitaceae & & & \\
\hline 134 & Cissus repanda (Wight \& Arn.) Vahl & $\mathrm{Cl}$ & HS & Very common \\
\hline \multirow[t]{3}{*}{135} & Tetrastigma planicaule (Hook.f.) Gagnep. & $\mathrm{Cl}$ & HS & Common \\
\hline & MONOCOTY & EDONS & & \\
\hline & Amaryllidaceae & & & \\
\hline 136 & Crinum lorifolium Roxb. ex Ker Gawl & $\mathrm{H}$ & $\mathrm{RS}$ & Rare \\
\hline
\end{tabular}


A. Kar et al. 131

\begin{tabular}{|c|c|c|c|c|}
\hline SI No & Name of the plants & Habit & Habitat & Distribution \\
\hline & Araceae & $\mathrm{H}$ & & \\
\hline 137 & Amorphophallus bulbifer (Roxb.) Blume & $\mathrm{H}$ & RS & Common \\
\hline 138 & $\begin{array}{l}\text { Amorphophallus paeoniifolius (Dennst.) } \\
\text { Nicolson }\end{array}$ & $\mathrm{H}$ & HS & Rare \\
\hline 139 & Colocasia_esculenta (L.) Schott & $\mathrm{H}$ & RS & Very common \\
\hline 140 & Lasia spinosa $(\mathrm{L})$ Thwaites & $\mathrm{H}$ & SS & Rare \\
\hline 141 & Monstera deliciosa Liebm. [Photo : 18] & EP & $\mathrm{F}$ & Rare \\
\hline 142 & Philodendron perplexum G.S Bunting & $\mathrm{H}$ & $\mathrm{F}$ & Rare \\
\hline 143 & Syngonium auritum (L.) Schott & $\mathrm{H}$ & $\mathrm{F}$ & Rare \\
\hline \multirow[t]{2}{*}{144} & Typhonium trilobatum (L.) Schott & $\mathrm{H}$ & HS & Rare \\
\hline & Arecaceae/ Palmae & & & \\
\hline 145 & Areca catechu L. & PA & HS & Common \\
\hline 146 & Calamus erectus Roxb. & $\mathrm{CA}$ & SS & Rare \\
\hline 147 & Calamus flagellum Griff. ex Mart. & $\mathrm{CA}$ & RB & Rare \\
\hline \multirow[t]{2}{*}{148} & Calamus tenuis Roxb. & $\mathrm{CA}$ & RB & Common \\
\hline & Asparagaceae & & & \\
\hline 149 & Dracaena angustifoli (Medik.) Roxb. [Photo 7] & S & $\mathrm{F}$ & Rare \\
\hline \multirow[t]{2}{*}{150} & Peliosanthes teta Andrews [Photo 1] & $\mathrm{H}$ & $\mathrm{F}$ & Very rare \\
\hline & Colchicaceae & & & \\
\hline \multirow[t]{2}{*}{151} & Gloriosa superba L. & $\mathrm{H}$ & HS & Very rare \\
\hline & Commelinaceae & & & \\
\hline 152 & Commelina benghalensis $\mathrm{L}$. & $\mathrm{H}$ & RS & Common \\
\hline 153 & Commelina diffusa Burm.f. & $\mathrm{H}$ & RS & Common \\
\hline 154 & Cyanotis cristata (L.) D.Don & $\mathrm{H}$ & $\mathrm{RS}$ & Common \\
\hline \multirow[t]{2}{*}{155} & Murdannia nudiflora (L.) Brenan & $\mathrm{H}$ & SS & Common \\
\hline & Cyperaceae & & & \\
\hline 156 & Bulbostylis barbata (Rottb.) C.B. Clarke & SE & GL & Common \\
\hline 157 & Carex indica $\mathrm{L}$. & SE & GL & Common \\
\hline 158 & Cyperus compressus L. & SE & GL & Common \\
\hline 159 & Cyperus digitatus Roxb. & SE & GL & Common \\
\hline 160 & Cyperus imbricatus Retz. & SE & GL & Common \\
\hline 161 & Cyperus iria $\mathrm{L}$. & SE & GL & Common \\
\hline 162 & Cyperus dubius Rottb. & SE & GL & Common \\
\hline 163 & Eleocharis dulcis (Burm.f.) Trin. ex Hensch. & SE & GL & Common \\
\hline 164 & Eleocharis spiralis (Rottb.) Roem. \& Schult. & SE & $\mathrm{RS}$ & Common \\
\hline 165 & $\begin{array}{l}\text { Fimbristylis dichotoma subsp. podocarpa } \\
\text { (Nees) T.Koyama . }\end{array}$ & SE & RS & Common \\
\hline 166 & Bolboschoenus maritimus (L.) Palla. & $\mathrm{SE}$ & GL & Common \\
\hline 167 & Isolepis setacea (L.) R.Br. & SE & GL & Common \\
\hline 168 & Scleria rugosa R. Br. & SE & GL & Common \\
\hline
\end{tabular}



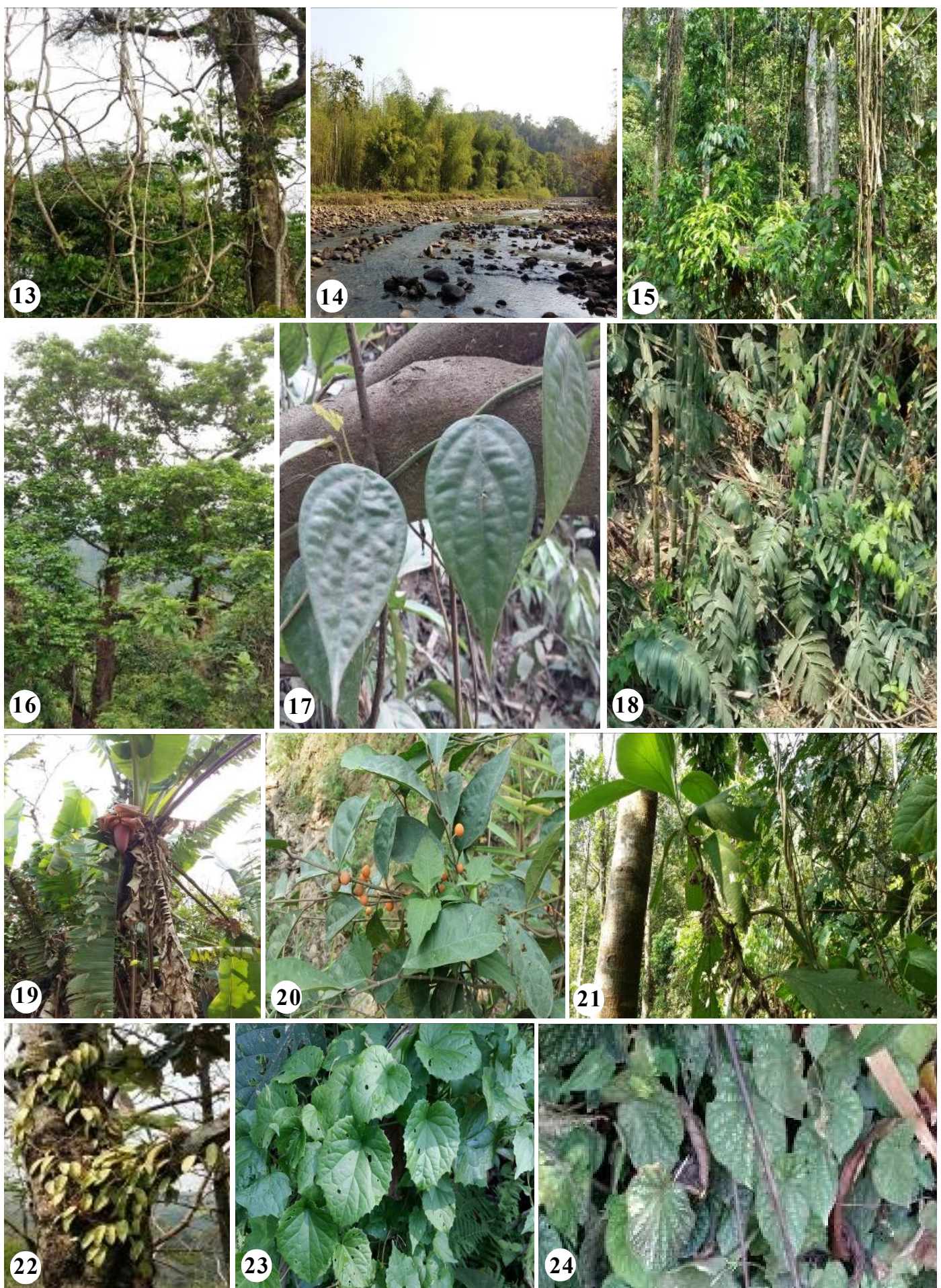

PLATE - II. 13. Lianas in BWS; 14. Riverine forest of BWS; 15. Evergreen forest of BWS; 16. Bischofia javanica; 17. Piper berembunse; 18. Monstera deliciosa; 19. Musa sanguinea; 20. Ficus tinctoria; 21. Gomphostemma parviflorum; 22. Hoya acuminata; 23. Natsiatum herpenticum; 24. Begonia thomsonii 
A. Kar et al. 133

\begin{tabular}{|c|c|c|c|c|}
\hline SI No & Name of the plants & Habit & Habitat & Distribution \\
\hline & Dioscoreaceae & & & \\
\hline \multirow[t]{2}{*}{169} & Dioscorea hispida Dennst. & $\mathrm{Cl}$ & $\mathrm{F}$ & Common \\
\hline & Marantaceae & & & \\
\hline \multirow[t]{2}{*}{170} & Clinogynae dichotoma (Roxb.) Salisb. & $\mathrm{S}$ & RB & Common \\
\hline & Musaceae & & & \\
\hline \multirow[t]{2}{*}{171} & Musa sanguinea Hook.f. [Photo 19] & $\mathrm{H}$ & $\mathrm{F}$ & Common \\
\hline & Orchidaceae & & & \\
\hline 172 & Aerides multiflora Roxb. & EP & $\mathrm{F}$ & Common \\
\hline 173 & Aerides odorata Lour. & EP & $\mathrm{F}$ & Common \\
\hline 174 & Cymbidium aloifolium (L.) Sw. [Photo 4] & EP & $\mathrm{F}$ & Common \\
\hline 175 & Dendrobium lituiflorum Lindl. [Photo 12] & EP & $\mathrm{F}$ & Rare \\
\hline \multirow[t]{2}{*}{176} & Liparis elliptica Wight. [Photo 2] & $\mathrm{H}$ & GL & Very rare \\
\hline & Pandanaceae & & & \\
\hline \multirow[t]{2}{*}{177} & Pandanus foetidus Roxb. & $\mathrm{H}$ & $\mathrm{F}$ & Rare \\
\hline & Poaceae/ Gramineae & & & \\
\hline 178 & Bambusa vulgaris Schrad. & B & $\mathrm{F}$ & Common \\
\hline 179 & Chrysopogon aciculatus (Retz.) Trin. & G & $\mathrm{F}$ & Common \\
\hline 180 & $\begin{array}{l}\text { Dendrocalamus hamiltonii Nees \& Arn. ex } \\
\text { Munro }\end{array}$ & B & $\mathrm{F}$ & Rare \\
\hline 181 & Digitaria ciliaris (Retz.) Koeler & $\mathrm{G}$ & $\mathrm{RS}$ & Common \\
\hline 182 & Eragrostis atrovirens (Desf.) Trin. ex Steud. & $\mathrm{G}$ & GL & Common \\
\hline 183 & Panicum brevifolium L. & $\mathrm{G}$ & GL & Common \\
\hline 184 & Saccharum spontaneum L. & $\mathrm{G}$ & GL & Common \\
\hline 185 & Setaria pumila (Poir.) Roem. \& Schult. & $\mathrm{G}$ & GL & Common \\
\hline 186 & Microstegium fasciculatum (L.) Henrard & G & GL & Common \\
\hline \multirow[t]{2}{*}{187} & Themeda villosa (Lam.) A. Camus & G & RS & Common \\
\hline & Smilacaceae & & & \\
\hline \multirow[t]{2}{*}{188} & Smilax glabra Roxb. & $\mathrm{Cl}$ & $\mathrm{F}$ & Common \\
\hline & Zingiberaceae & & & \\
\hline 189 & Alpinia roxburghii Sweet & $\mathrm{H}$ & HS & Rare \\
\hline 190 & Curcuma aromatica Salisb. & $\mathrm{H}$ & $\mathrm{F}$ & Rare \\
\hline
\end{tabular}

Aglaia is the dominant genera represented with 4 species followed by Derris, Grewia, Ailanthus and Dysoxylum each with 3 species, Garuga and Dillenia each with 2 species. Among the monocotyledons Cyperus is the most dominant genus represented by 5 species followed by Calamus with 3 species and Aerides, Commelina and Amorphophallus with 2 species each.

With regard to the habitat preference of these 188 plant species, 106 species are from inside the forest, 37 species from the road-sides, nine species from the stream margins, five from river banks, 12 from hill slopes and 19 species from the grassland areas.

It is interesting to note that Liparis elliptica with only four plants in one population were recorded from the whole study area. Among herbs, Peliosanthes teeta, among shrubs Gomphostemma parviflorum, Ardisia paniculata, Ixora rangonensis, Morinda 


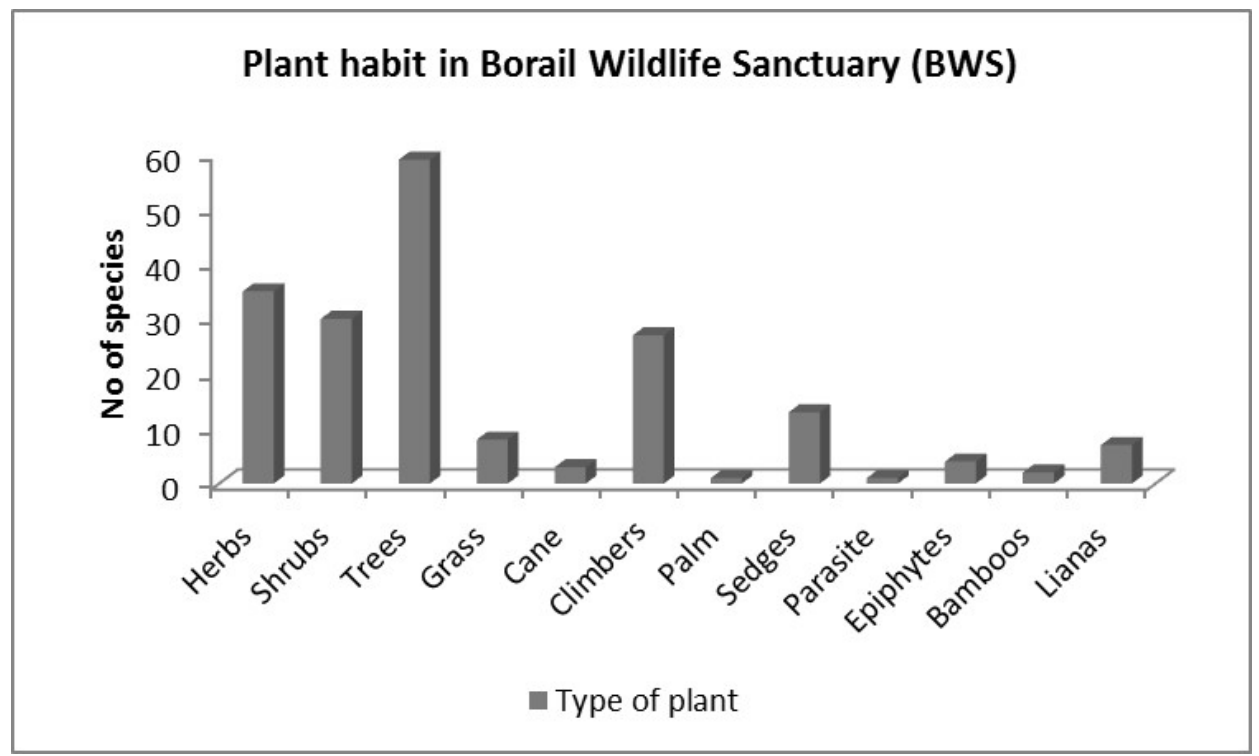

Figure 2. Plant habit in Borail Wildlife Sanctuary (BWS)

angustifolia, Dracaena angustifolia; among climbers Piper berembunse, Todalia asiatica and among trees Bischofia javanica, Litsea monopetala, Saraca asoca, Mangifera sylvatica and Dillenia pentagyna were very rare and were located in restricted pockets.

was observed that, there is severe anthropogenic pressure on the sanctuary and that is now in an alarming cause for the decline of the reserve's species diversity. Major problem of the sanctuary is deforestation due to construction works of East-West Corridor which is passing through East block of the sanctuary. I have missed out to relocate some species from the same sites which were observed during my previous study as these species were vanished from particular site due to road widening activities. Expansion of Areca nut plantation, Tea plantation, Teak plantation and cultivation of black pepper and vegetables in fringe areas are also threat for the sanctuary. Extraction of important species particularly Homalomena aromatica, Bamboos, Dalchini, Amla etc. from the forest areas is a matter of concern. Concerned department and local civil society should take care on these issues to protect the valuable plant resources and wildlife of the sanctuary.

\section{Acknowledgements}

Authors are thankful to the Chief Conservator of Forests, Southern Assam Circle, Assam for granting permission to carry out the study in Borail Wildlife Sanctuary. The first author (A.Kar) acknowledges the Department of Biotechnology (DBT), Govt. of India, New Delhi for financial support to undertake this study under the DBT-Foldscope programme.

\section{LITERATURE CITED}

The Plant List 2013. The Plant List. Version 1.1. http://www.theplan-tlist.org/. [Accessed on: November 2018].

APG III. 2009. An update of the Angiosperm Phylogeny Group clas-sification for the orders and families of flowering plants: APG III. Bot. J. Linn. Soc. 161: $105-121$. https:// doi.org/10.1111/j.10958339.2009.00996.x 
Barbhuiya, H.A \& Singh, S.K. 2012. Liverwort and Hornwort of Borail Wild Life Sanctuary, Assam, India. Archive for Bryology 134: 1 - 12.

Baruah, P.P. \& Baruah, C.K. 2000.Study of the hydrophytes flora of Kaziranga National Park, Assam, India. Ann. For. 8(2): 170 - 178.

Baruah, P.P. \& Baruah, C.K. 2007.Vegetational characteristics of grassland of Kaziranga National Park, Assam, India. In : P. C. Trivedy (ed.), Global Biodiversity: status and conservation. Pointer Publication, Joipur, Pp. $210-219$.

Baruah, C.K.; Sharma G.C.; Bezbarua, P. \& Phukan U. 2003. Biodiversity status in Manas Biosphere reserve. Report submitted to Ministry of Enviroment \& Forests, Govt. of India, Pp. $1-40$.

Begum, S.S.; Roy, H.; Nath, M. \& Borthakur S.K. 2011. A sketch of the flora of Nameri National Park, Assam:II. Phytogeography, Pleione 5(1): 10 - 22.

Bharali, P. \& Borua, P.K. 2003. Diversity of orchid flora of Dibru-Saikhowa National Park and Biosphere Reserve, Assam. In: P.P. Baruah (ed.), Biodiversity of Eastern Himalayan Protected Areas. Dept. of Botany, Handique Girls' College, Guwahati. Pp. $47-57$.

Bora, A. \& Bhattacharyya, D. 2017. Phytodiversity of Barail Wildlife Sanctuary, Assam, India: field based observations- I. Trees and lianas. Check List 13(6): 1037 - 1053.

Bora, A.; Devi, M. \& Bhattacharyya, D. 2017. Grasses and bamboos of Barail Wildlife Sanctuary in Assam, India. Pleione 11(2): 440 - 454.

Buragohain, R. \& Swargiari, B.N. 2016. Diversity and conservation of Ficus Linnaeus (Moraceae) in Chakrashila Wildlife Sanctuary, Kokrajhar District of Assam, India. Pleione 10(2): $302-309$

Bujarbarua, P. \& Sarma, S.K. 2006. A note on the diversity of family Poaceae in Gibbon Wildlife Sanctuary, Assam, India. J. Econ. Taxon. Bot. 30(1): $1-5$.

Champion, H.G. \& Seth, S.K. 1968. A revised survey of forest types of India, Govt. of India Press, Delhi

Chowdhury, S. 2005. Assam's Flora (Present status of vascular plants), Assam Science Technology and Environment Council, Guwahati

Deori, C. \& Talukdar, S.R. 2015. Floristic Diversity of Barnadi Wildlife Sanctuary, Assam. Nelumbo 57(2): 19 - 28.

Dey, S.; Lakhar, B.P.; Das, J.P.; Nath, N.K. \& Brahma, N. 2007. Orchid diversity in Manas National Park, Assam. J. Orch. Soc. India 21(1-2): 65 - 68.

Dutta, A.K.; Dutta, T.K. \& Gupta, K.K. 1974. A tentative accounting of the forest flora of North Cachar Hills and Borail Range. Indian For. 100: 60 - 76.

Gogoi, K.; Borah, R.L. \& Sharma, G.C. 2009. Orchid flora of Joypur Reserve Forest of Dibrugarh district of Assam, India. Pleione 3(2): 135 - 147.

Gogoi, K.; Borah, R.L. \& Sharma, G.C. 2010. Orchid flora of Dibru-Saikhowa National Park and Biosphere Reserve, Assam, India. Pleione, 4 (1): 124 - 134.

Gogoi, K. 2005. The genus Dendrobium in Dibru-Saikhowa National park and Biosphere Reserve. J. Orch. Soc. India 19(1-2): 17 - 25.

Hajra, P.K. \& Jain, S.K. 1996. Botany of Kaziranga and Manas. Surya International Publications, Dehra Dun. 
Hussain, M.M. 2015. Technical Report: An Inventory Survey for the Forested IBAs in Barak Valley, Assam. (Supported by IBCN Small Grants 2014). Centre for Biodiversity and Natural Resource Conservation Department of Ecology \& Environmental Science Assam University, Silchar, Assam

IPNI 2012. The International Plant Names Index. http://www.ipni.org. Accessed on: 09-112018.

Jain, S.K. \& Rao, R.R. 1977. A Hand Book of Field and Herbarium Technique. Today \& Tomorrow's Publication, New Delhi.

Jain, S. \& Hajra, P. 1975. On the botany of Manas Wildlife Sanctuary in Assam. Bull. Bot. Surv. India 17: $75-86$.

Kanjilal, U.N.; Kanjilal, P.C.; Das, A. \& De, R.N. 1934 - 1940. Flora of Assam. Vol. I-V Govt Press, Shillong, India

Kar, A.; Borah, R. Goswami, N. K \& Saharia, D. 2015. Inventorization of vascular plant diversity in Amchang Wildlife Sanctuary, Kamrup Metro district, Assam. Indian J. Appl. Res. 5(2): $48-60$

Kar, A.; Boruah, D.; Goswami, N. K \& Saharia, D. 2018. Angiospermic flora of Borail Wildlife Sanctuary (BWS), in Assam, India: First report. Pleione 12(2): 193 - 207.

Konwar, P.; Kakati, S.M. \& Saikia, P.K. 2009. Abundance of food plant species and food habits of Rhinoceros unicornis Linn. in Pobitora Wildlife Sanctuary, Assam, India. $J$. Threat. Taxa 1(9): 457 - 460.

Nath, S.K. 2012. Aquatic macrophytes of Laokhowa Wildlife Sanctuary, Assam, India. Intn. J. Eng. Res. App. 2: 1911 - 1913.

Sarkar, M. \& Devi, A. 2015. Assessment of plant diversity in Hollongapar Gibbon Wildlife Sanctuary, Assam, Northeast India. Indian For. 141(6): 648 - 659.

Talukdar, S.R. \& Deori, C. 2017. Floristic Diversity of Laokhowa Wildlife Sanctuary, Assam, India. Nelumbo 59(2): $168-177$.

Tropicos. 2017. Tropicos. http://www.tropicos.org/Home.aspx. Acces-sed on:[June 2019].

WWF 2018. We need to safeguard our forest (Article). WWF- India. 